\title{
Associação entre diabetes mellitus e doenças oculares em pessoas com deficiência visual
}

\author{
Association between diabetes mellitus and eye diseases in people with visual impairment
}

Asociación entre diabetes mellitus y enfermedades oculares en personas con discapacidad visual

\author{
Evandro Scarso de Brito' ๑; Maria Helena Pinto" $\odot$; Denise Beretta" $\odot$; Lilian Cristina de Castro Rossill ${ }^{\oplus}$; \\ Thiare Júnia de Francisco Brito ${ }^{\prime V} \odot$; Rildo César Nunes Czornyl'I! ๑
}

'Secretaria Municipal de Saúde de São José do Rio Preto, São José do Rio Preto São José do Rio Preto, SP, Brasil; "Faculdade de Medicina de
São José do Rio Preto, São José do Rio Preto, SP, Brasil; "'União das Faculdade dos Grandes Lagos, São José do Rio Preto, SP, Brasil;
"Associação dos Pais e Amigos dos Excepcionais de São José do Rio Preto, São José do Rio Preto, SP, Brasil

\section{RESUMO}

Objetivo: verificar associação entre diabetes mellitus e doenças oculares em pessoas com deficiência visual. Método: estudo transversal com 51 pessoas com diabetes e deficiência visual, em um centro de reabilitação visual do interior paulista, que participaram de entrevista estruturada, em 2018. Utilizou-se os testes: Kolmogorov Smirnov, Regressão de Poisson, Regressão de Logística Binária, e Correlação de Spearman. Resultados: a maioria das pessoas era cega e relatou que a retinopatia diabética, o glaucoma e a catarata foram causa da deficiência visual; com tempo de diagnóstico do diabetes acima de 109 meses. A catarata apresentou um nível de correlação baixa $(r=0,280$ e $p=0,047)$, e a retinopatia diabética um nível de correlação moderada $(r=0,565$ e $p=0,000)$, considerando o tempo de diagnóstico do diabetes. Conclusão: associação estatisticamente significante entre o tipo de diabetes e a retinopatia, e correlação estatisticamente significante entre o tempo de diagnóstico do diabetes, a catarata e a retinopatia diabética.

Descritores: Diabetes Mellitus; Catarata, Retinopatia Diabética, Glaucoma.

\begin{abstract}
Objective: to verify the association between diabetes mellitus and eye diseases in people with visual impairment. Method: this cross-sectional study involved 51 people with diabetes and visual impairment at a Visual Rehabilitation Center in São Paulo, who participated in a structured interview in 2018. The tests used were: Kolmogorov Smirnov, Poisson Regression, Binary Logistic Regression, and Spearman Correlation. Results: most participants were blind, reported that diabetic retinopathy, glaucoma and cataracts were the causes of their visual impairment, and had been diagnosed with diabetes over 109 months earlier. Cataract returned a low level of correlation with time with diagnosis of diabetes $(r=0.280$ and $p=0.047)$, and diabetic retinopathy, moderate correlation $(r=0.565$ and $p=0.000)$. Conclusion: a statistically significant association was found between type of diabetes and retinopathy, and statistically significant correlations between the time diagnosed with diabetes, cataracts and diabetic retinopathy.
\end{abstract}

Descriptors: Diabetes Mellitus; Cataract, Diabetic Retinopathy, Glaucoma.

\section{RESUMEN}

Objetivo: verificar la asociación entre diabetes mellitus y enfermedades oculares en personas con discapacidad visual. Método: este estudio transversal involucró a 51 personas con diabetes y discapacidad visual en un Centro de Rehabilitación Visual en São Paulo, quienes participaron en una entrevista estructurada en 2018.Las pruebas utilizadas fueron: Kolmogorov Smirnov, Regresión de Poisson, Regresión Logística Binaria y Spearman Correlación. Resultados: la mayoría de los participantes eran ciegos, informaron que la retinopatía diabética, el glaucoma y las cataratas eran las causas de su discapacidad visual y habían sido diagnosticados con diabetes más de 109 meses antes. La catarata devolvió un bajo nivel de correlación con el tiempo con el diagnóstico de diabetes $(r=0,280$ yp $=0,047)$ y la retinopatía diabética, correlación moderada $(r=0,565$ yp $=0,000)$. Conclusión: se encontró asociación estadísticamente significativa entre tipo de diabetes y retinopatía, y correlaciones estadísticamente significativas entre el tiempo de diagnóstico de diabetes, cataratas y retinopatía diabética.

Descriptores: Diabetes Mellitus; Catarata, Retinopatía Diabética, Glaucoma.

\section{INTRODUÇÃO}

A deficiência visual (DV) é definida pela perda parcial ou total da visão, sendo classificada em baixa visão ou visão subnormal e cegueira; e suas causas podem ser congênita ou adquirida ${ }^{1}$.

As principais causas da DV em adultos e idosos estão associadas ao envelhecimento da população. No Brasil, como a população apresenta uma das maiores taxas de aumento da expectativa de vida entre os países mais populosos do mundo, temos também a expectativa do aumento da prevalência de tais causas, principalmente o diabetes mellitus (DM) ${ }^{2}$.

O glaucoma e a catarata são patologias frequentes e precoces em pessoas com diabetes ${ }^{3}$. A retinopatia diabética (RD) é a principal e mais prevalente complicação microvascular ${ }^{4}$, que atinge mais de um terço das pessoas com diabetes, sendo considerada a principal causa de perda de visão em adultos em idade ativa 5 . 
Considerando a alta prevalência, gravidade e relevância do DM e da DV no Brasil e no mundo, e a escassez de artigos na literatura relacionada sobre essa associação ${ }^{6}$, este estudo teve como objetivo verificar a associação entre o DM e doenças oculares em pessoas com deficiência visual de um Centro de Reabilitação Visual.

\section{REVISÃO DE LITERATURA}

O DM é considerado um problema de saúde pública mundial, crescente e importante, independente do grau de desenvolvimento dos países ${ }^{7}$. É caracterizado pela concentração de glicose aumentada no sangue podendo ser consequente da secreção insuficiente de insulina, da resistência à ação da insulina, ou a ambos ${ }^{8}$.

Estima-se que em 2045, o número de pessoas com DM, com idade igual ou superior a 20 anos, aumentará de 449,3 para 691,6 milhões. No Brasil, em 2017, o DM acometeu 12,5 milhões de pessoas com idade entre 20 e 79 anos, com projeção de 21,8 milhões em 2045. Aproximadamente quatro milhões de pessoas com idade entre os 20 e 79 anos morreram de DM em 2017 no mundo, equivalendo a uma morte a cada oito segundos 5 .

As pessoas com diabetes possuem 30 vezes mais chances de tornarem-se cegos, do que pessoas que não possuem a doença ${ }^{2}$, e caso o DM não seja tratado ou mal controlado podem desenvolver mais complicações ${ }^{7}$.

De acordo com as Diretrizes da Sociedade Brasileira de Diabetes (SBD), no mundo há 93 milhões de pessoas com diabetes que possuem algum grau de RD, enquanto no Brasil aproximadamente quatro milhões de pessoas possuem a doença'.

O glaucoma é considerado a segunda maior causa de cegueira no mundo, com incidência estimada de 1 a $2 \%$ da população em geral, aumentando esse percentual após os 40 anos de idade². Mundialmente estima-se que 95 milhões de pessoas sejam afetadas pela catarata ${ }^{9}$. No Brasil, em 2015, 350.000 pessoas ficaram cegas por catarata, com aumento de $20 \%$ dos casos novos a cada ano ${ }^{2}$.

O último Censo Demográfico realizado no Brasil em 2010 apontou que mais de 45 milhões de brasileiros (23,9\% da população total) possui pelo menos uma deficiência, sendo a DV a mais prevalente $(18,6 \% \text { da população brasileira })^{10}$. No mundo, estima-se que mais de 407 milhões de pessoas possui algum tipo de DV, sendo que 188 milhões possuem comprometimento visual leve, mais de 216 milhões possuem DV moderada a grave, e 36 milhões são cegas ${ }^{3}$.

\section{MÉTODO}

Estudo transversal, descritivo, de caráter quantitativo, desenvolvido em um centro de reabilitação visual do interior paulista. A instituição atende pessoas de todas as fases do ciclo de vida que possuem DV (baixa visão e cegueira), referenciada pelos estabelecimentos vinculados ao Sistema Único de Saúde (SUS); possuí 241 matriculados, 113 são adultos ou idosos, e desses, 62 com diabetes.

A população do estudo foi constituída por todas as pessoas com diabetes e deficiência visual, adultas ou idosas, matriculadas na instituição. Os critérios de inclusão foram: ter idade igual ou superior a 18 anos e estar frequentando a instituição. Assim, foram excluídas onze pessoas, sendo que seis por não estarem mais frequentando a instituição e cinco por não terem aceitado participar da entrevista, ficando a amostra constituída de 51 pessoas.

A coleta de dados foi realizada de junho a agosto de 2018 pelo pesquisador, que agendou um encontro com as pessoas que aceitaram o convite de participar da pesquisa. Cada um dos participantes, após apresentação do objetivo e delineamento da pesquisa, assinou o Termo de Consentimento Livre e Esclarecido, que foi lido através de um dispositivo portátil e independente que amplia e lê em alto som materiais impressos para pessoas com DV.

Em seguida, realizou-se uma entrevista estruturada de forma individualizada e privativa, com duração média de 40 minutos, seguindo um instrumento de coleta de dados elaborado pelo pesquisador que contemplavam as seguintes variáveis: sexo, idade, tipo da DV, causa da DV, presença das doenças oculares - glaucoma, catarata e RD, tempo da DV, tipo do DM e tempo de diagnóstico do DM.

Para análise dos dados, utilizou-se o Software SPSS Statistics, versão 23, atreladas às funcionalidades da ferramenta Excel $^{\circledR}$, versão 2016. Inicialmente foi realizada uma análise descritiva das variáveis, sendo as quantitativas descritas em médias e desvios padrão; e as qualitativas, em frequências simples e percentuais. Testou-se a normalidade pelo teste de Kolmogorov Smirnov. Utilizou-se a regressão de Poisson para analisar a dependência de variáveis dicotômicas quando cruzadas com variáveis dicotômicas $(2 \times 2)$ ou contínuas, a regressão de Logística Binária para cruzamento multivariado, e correlação de Spearman, para verificar a correlação entre as variáveis "tempo de diagnóstico do DM" e as "doenças oculares". Os testes contemplam erro alfa de $5 \%$ e confiabilidade de $95 \%$, assim, as associações foram consideradas estatisticamente significativas quando $p<0,05$. 
Este estudo foi submetido e aprovado pelo Comitê de Ética e Pesquisa (CEP) da instituição, sob parecer no 2.595.328, atendendo as exigências legais para estudo envolvendo seres humanos, conforme a Resolução 466/12 do Conselho Nacional de Ética em Pesquisa.

\section{RESULTADOS E DISCUSSÃO}

Foram entrevistadas 51 pessoas com diabetes e deficiência visual, com predominância do sexo masculino (58\%), idade igual ou superior a 60 anos (43,1\%), idade média de 54 anos e desvio padrão de 16,3 anos. Esses resultados foram semelhantes ao estudo realizado no Distrito Federal com pessoas com diabetes, o qual também constatou predomínio do sexo masculino, idade entre 60 e 70 anos, que possuíam alteração visual ${ }^{11}$.

Entretanto, estudos internacionais retratam uma realidade diferente. Em pesquisa realizada na Hungria, as pessoas com diabetes, com idade igual ou superior a 50 anos, eram a maioria do sexo feminino, 13,80\% possuía algum tipo de DV e 0,90\% eram cegos $^{12}$. No país de Gana, em ambiente hospitalar, identificou-se que das pessoas com diabetes que frequentavam a instituição, predominou o sexo feminino, 18,40\% possuía algum tipo de DV e 5,80\% eram cegos ${ }^{13}$. No México, estudo realizado com pessoas com diabetes, também acima de 50 anos, mostrou que a maioria era do sexo feminino, $14,90 \%$ possuía algum tipo de DV, e $1,50 \%$ era cega ${ }^{14}$.

Além disso, observou-se neste estudo que a maioria das pessoas (72,55\%) era cega e a catarata prevaleceu tanto nos cegos (59,46\%) quanto nos com baixa visão (57,14\%); o glaucoma prevaleceu nos cegos (51,35\%); e a RD não prevaleceu em mais da metade dos cegos e nos com baixa visão. As doenças oculares (RD, glaucoma e catarata) foram à causa da DV relatadas pela maioria das pessoas (62,75\%), com associação estatisticamente significante entre a causa e os tipos da DV $(p=0,000)$ (Tabela 1). Esses dados vão ao encontro ao estudo realizado no estado da Bahia ${ }^{15}$, no qual $53,90 \%$ das pessoas idosas com diabetes possuíam a complicação visual; e pesquisa realizada no Sul da Índia, que acompanhou as pessoas com diabetes durante quatro anos, e identificou a incidência de DV (21,70\%), sendo que leve $(18,30 \%)$ e moderado $(3,40 \%)^{16}$.

TABELA 1: Distribuição de doenças oculares e a causa da DV, segundo os tipos da DV, referidas pelas pessoas com diabetes. São José do Rio Preto, SP, Brasil, 2018.

\begin{tabular}{|c|c|c|c|c|c|c|c|}
\hline \multirow[t]{2}{*}{ Variáveis } & \multicolumn{2}{|c|}{ Baixa Visão } & \multicolumn{2}{|c|}{ Cego } & \multicolumn{2}{|c|}{ Total } & \multirow[b]{2}{*}{$\mathbf{p}$} \\
\hline & $\mathbf{n}$ & $\%$ & $\mathbf{n}$ & $\%$ & $\mathbf{n}$ & $\%$ & \\
\hline \multicolumn{8}{|l|}{ Catarata } \\
\hline Não & 6 & 42,86 & 15 & 40,54 & 21 & 41,18 & \multirow{2}{*}{$0,923^{* *}$} \\
\hline Sim & 8 & 57,14 & 22 & 59,46 & 30 & 58,82 & \\
\hline \multicolumn{8}{|l|}{ Glaucoma } \\
\hline Não & 9 & 64,29 & 18 & 48,65 & 27 & 52,94 & \multirow{2}{*}{$0,266 * *$} \\
\hline Sim & 5 & 35,71 & 19 & 51,35 & 24 & 47,06 & \\
\hline \multicolumn{8}{|c|}{ Retinopatia Diabética } \\
\hline Não & 8 & 57,14 & 19 & 51,35 & 27 & 52,94 & \multirow{2}{*}{$0,549 * *$} \\
\hline Sim & 6 & 42,86 & 18 & 48,65 & 24 & 47,06 & \\
\hline \multicolumn{8}{|c|}{ Causa da Deficiência Visual } \\
\hline Doenças Oculares* & 8 & 57,14 & 24 & 64,86 & 32 & 62,75 & \multirow{3}{*}{$0,000 * *$} \\
\hline Outras & 6 & 42,86 & 13 & 35,14 & 19 & 37,25 & \\
\hline TOTAL & 14 & 27,45 & 37 & 72,55 & 51 & 100,00 & \\
\hline
\end{tabular}

*Doenças Oculares: RD, Glaucoma e Catarata

**Aplicado teste de Regressão de Poisson a $p<0,05$

No presente estudo, o glaucoma não predominou em nenhum dos tipos de DM, sendo que $52,17 \%$ das pessoas com diabetes tipo 1 e 53,57\% do tipo 2 não tinham a doença; como também não prevaleceu na maioria da amostra estudada (52,94\%). A catarata prevaleceu na maior parte das pessoas (58,82\%), como também em ambos os tipos de DM (65,11\% no tipo 1 e 53,57\% no tipo 2). A RD prevaleceu na maioria das pessoas com diabetes tipo 1 (78,26\%), com uma associação estatisticamente significante (teste de Regressão de Poisson a $p<0,05$ ) entre o tipo de DM e a presença da RD $(p=0,008)$.

Ademais, estudo com pessoas com diagnóstico de catarata na Venezuela identificou o DM como a doença associada mais prevalente ${ }^{17}$. A relação entre catarata e o DM também foi relatada pela maioria das pessoas de um estudo realizado no estado da Paraíba. O DM tipo 2 provoca mudanças na fisiologia ocular e a opacificação do cristalino, tornando a catarata, a principal causa de cegueira adquirida no Brasil ${ }^{18}$.

Estudo realizado no Nepal, cujo objetivo era verificar a associação entre DM e glaucoma, identificou em sua amostra que $27,40 \%$ das pessoas com diabetes tipo 2 possuíam a doença ${ }^{19}$. Em um estudo de coorte retrospectivo, com 
período de acompanhamento de 10 anos, realizado na Coréia do Sul demonstrou que o desenvolvimento do glaucoma foi significativamente maior em pessoas com DM, comparado com os que não possuíam a doença, independentemente da idade e $\operatorname{sexo}^{20}$. No presente estudo, o glaucoma prevaleceu nos cegos e não predominou em nenhum tipo de DM, como também não prevaleceu na maioria da amostra estudada.

Apesar da RD não ter prevalecido em mais da metade dos cegos e naqueles com baixa visão, a maioria das pessoas com diabetes tipo 1 possuía a doença, com uma associação estatisticamente significante, assim como no estudo realizado em Portugal, o qual constatou que as pessoas com DM tipo 1 apresentavam prevalências da RD superiores aos do tipo 2 com diferenças estatisticamente significativas ${ }^{21}$.

As pessoas com DM tipo 1 e com maior tempo de DM possuem um risco aumentado para $R^{22}$ e consequentemente para a cegueira ${ }^{2}$, corroborando achados deste estudo, uma vez que o tempo da DV e os tipos de DM tiveram uma associação estatisticamente significante. A maioria das pessoas com diabetes tipo 1 e tipo 2 descobriu o DM há mais de 109 meses, entretanto, desses, apenas as pessoas com diabetes tipo 1 relataram que se tornaram deficientes visuais há menos de 108 meses, inferindo-se assim, que nessas pessoas, o DM foi um dos determinantes para o desenvolvimento das doenças oculares e consequentemente para a DV.

Destaca-se que $91,3 \%$ das pessoas com diabetes tipo 1 e 53,58\% tipo 2 referiram que descobriram o DM há mais de 109 meses. Entretanto, ao analisar o tempo de DV, a maior parte das pessoas com diabetes tipo 1 (69,56\%) relatou que se tornaram deficientes visuais há menos de 108 meses; nas pessoas com diabetes tipo 2 predominou o tempo da DV acima de 109 meses (60,71\%). Observa-se também uma associação estatisticamente significante $(p=0,022)$ entre o tempo da DV e os tipos de DM (Tabela 2).

TABELA 2: Distribuição do tempo de diagnóstico do DM e o tempo da DV, segundo o tipo de DM. São José do Rio Preto, SP, Brasil, 2018.

\begin{tabular}{lcccccccc}
\hline Variáveis & \multicolumn{1}{c}{ DM TIPO $\mathbf{1}$} & \multicolumn{2}{c}{ DM TIPO $\mathbf{2}$} & \multicolumn{2}{c}{ Total } \\
& $\mathbf{n}$ & $\mathbf{\%}$ & $\mathbf{n}$ & $\mathbf{\%}$ & $\mathbf{n}$ & $\mathbf{\%}$ & $\mathbf{p}$ \\
\hline Tempo de diagnóstico do DM & & & & & & & \\
Até 36 meses & 1 & 4,35 & 6 & 21,43 & 7 & 13,73 & \\
37 a 72 meses & 1 & 4,35 & 3 & 10,71 & 4 & 7,84 & \\
73 a 108 meses & - & 0,00 & 4 & 14,29 & 4 & 7,84 & $0,057^{*}$ \\
109 a 144 meses & 4 & 17,39 & 4 & 14,29 & 8 & 15,69 & \\
> 144 meses & 17 & 73,91 & 11 & 39,29 & 28 & 54,90 & \\
Tempo da DV & & & & & & & \\
Até 36 meses & 9 & 39,13 & 5 & 17,86 & 14 & 27,45 & \\
37 a 72 meses & 3 & 13,04 & 5 & 17,86 & 8 & 15,69 & \\
73 a 108 meses & 4 & 17,39 & 1 & 3,57 & 5 & 9,80 & $0,022^{*}$ \\
109 a 144 meses & 2 & 8,70 & 3 & 10,71 & 5 & 9,80 & \\
$>144$ meses & 5 & 21,74 & 14 & 50,00 & 19 & 37,25 & \\
TOTAL & $\mathbf{2 3}$ & $\mathbf{4 5 , 1 0}$ & $\mathbf{2 8}$ & $\mathbf{5 4 , 9 0}$ & $\mathbf{5 1}$ & $\mathbf{1 0 0 , 0 0}$ & \\
\hline
\end{tabular}

*Aplicado teste de Regressão Logística Binária a p<0,05

O tempo de diagnóstico do DM influencia a qualidade de vida das pessoas, uma vez que um tempo prolongado de DM aumenta o risco de complicações ${ }^{23}$. Pesquisa realizada no estado da Bahia mostrou que quanto maior o tempo de diagnóstico do DM, menor é a percepção na qualidade de vida das pessoas ${ }^{24}$. Um estudo epidemiológico transversal com 13.473 pessoas adultas com diabetes realizado na China identificou que a prevalência de RD está intimamente relacionada com a duração da DM e a idade de início do DM $(p<0,001)^{25}$. Em Goiás, pesquisa identificou que 51,4\% das pessoas foram diagnosticadas com DM há mais de dez anos ${ }^{26}$. Os resultados deste estudo mostram também esta realidade, uma vez que a partir de 109 meses de diagnóstico do DM, quanto maior o tempo de diagnóstico, maior a prevalência de todas as doenças oculares (Tabela 3).

Após 15 anos de DM aproximadamente $2 \%$ das pessoas com diabetes tornam-se cegos e $10 \%$ desenvolvem perda visual grave; e após 20 anos da doença, mais de $75 \%$ das pessoas possuem alguma forma de $\mathrm{RD}^{2}$. No presente estudo, observou-se que quanto maior o tempo de diagnóstico do DM, maior a prevalência de catarata e RD, sendo que estas se correlacionaram de forma estatisticamente significativa. Esse dado vai ao encontro do estudo realizado em Portugal, que identificou um tempo de evolução do DM significativamente superior em indivíduos com $\mathrm{RD}^{21}$.

Ao analisar a correlação entre o tempo de diagnóstico do DM e a presença das doenças oculares, através da Correlação de Spearman, identificou-se uma correlação estatisticamente significante com a catarata e a RD, sendo que a catarata apresentou um nível de correlação baixa ( $r=0,280$ e $p=0,047)$, e a RD um nível de correlação moderada $(r=0,565$ e $p=0,000)$. Já o Glaucoma não apresentou correção ( $r=-0,074$ e $p=0,608)$. 
TABELA 3: Distribuição das doenças oculares, segundo o tempo de diagnóstico do DM. São José do Rio Preto, SP, Brasil, 2018.

\begin{tabular}{|c|c|c|c|c|c|c|}
\hline \multirow[b]{2}{*}{ Variáveis } & \multicolumn{6}{|c|}{ Tempo de diagnóstico do DM - n (\%) } \\
\hline & Até 36 meses & $\begin{array}{l}37 \text { a } 72 \\
\text { meses }\end{array}$ & $\begin{array}{c}73 \text { a } 108 \\
\text { meses }\end{array}$ & $\begin{array}{c}109 \text { a } 144 \\
\text { meses }\end{array}$ & $\begin{array}{c}>144 \\
\text { meses }\end{array}$ & Total \\
\hline \multicolumn{7}{|l|}{ Glaucoma } \\
\hline Não & $4(57,14)$ & $3(75,00)$ & $2(50,00)$ & $3(37,50)$ & $15(53,57)$ & $27(52,94)$ \\
\hline Sim & $3(42,86)$ & $1(25,00)$ & $2(50,00)$ & $5(62,50)$ & $13(46,43)$ & $24(47,06)$ \\
\hline \multicolumn{7}{|l|}{ Catarata } \\
\hline Não & $3(42,86)$ & $3(75,00)$ & $4(100,00)$ & $2(25,00)$ & $9(32,14)$ & $21(41,18)$ \\
\hline Sim & $4(57,14)$ & $1(25,00)$ & $0(0,00)$ & $6(75,00)$ & $19(67,86)$ & $30(58,82)$ \\
\hline \multicolumn{7}{|l|}{ RD } \\
\hline Não & $7(100,00)$ & $3(75,00)$ & $4(100,00)$ & $4(50,00)$ & $9(32,14)$ & $27(52,94)$ \\
\hline Sim & $0(0,00)$ & $1(25,00)$ & $0(0,00)$ & $4(50,00)$ & $19(67,86)$ & $24(47,06)$ \\
\hline TOTAL & $7(13,73)$ & $4(7,84)$ & $4(7,84)$ & $8(15,69)$ & $28(54,90)$ & $51(100,00)$ \\
\hline
\end{tabular}

Autores relataram que em pessoas com idades entre 16 e 64 anos, no ano entre 2009-2010, a RD não era mais a principal causa de cegueira no Reino Unido e no País de Gales, correspondendo a $14,40 \%$ dos registros de cegueira em comparação com $17,70 \%$ no ano entre $1999-2000^{27}$. As razões subjacentes a essa mudança incluem a introdução de programas nacionais de rastreamento de RD no Reino Unido e no País de Gales e melhorias no controle glicêmico, assim como educação das pessoas com diabetes acerca de como gerenciar sua condição, e a retardar a progressão e possivelmente evitar complicações relacionadas a visão ${ }^{28}$.

\section{Limitações do estudo}

As limitações do estudo estão relacionadas ao local da coleta de dados, não sendo possível e viável o acesso aos dados de prontuários para confirmar as informações relatadas. $E$, por tratar-se de uma pesquisa transversal sem acompanhamento clínico ao longo do tempo, pode refletir possíveis vieses de recordação, uma vez que o paciente relata informações passadas de cunho subjetivo.

\section{CONCLUSÃO}

Evidenciou-se, neste estudo, uma associação estatisticamente significante entre o tipo de diabetes e a RD, a qual a maioria das pessoas com diabetes tipo 1 possuía essa doença ocular. $E$, uma correlação estatisticamente significante entre o tempo de diagnóstico do DM, a catarata e a RD, inferindo-se que quanto maior o tempo de DM maior é o risco de desenvolver essas doenças oculares.

O tempo da DV e os tipos de DM também foram estatisticamente relevantes, sendo que a maioria das pessoas com diabetes tipo 1 tornou-se deficiente visual após a descoberta do DM. Nota-se, portanto que o DM é um dos principais fatores de risco para o desenvolvimento das doenças oculares e para a DV, principalmente nas pessoas com diabetes tipo 1.

Assim, espera-se que o estudo contribua na atuação dos profissionais da saúde, especialmente os enfermeiros, na promoção de hábitos de vida mais saudáveis, com ações educativas, seja para prevenção do DM, ou prevenção de complicações da doença, assim como possibilitar reflexão crítica e o repensar nas práticas no atendimento às pessoas com diabetes. Para isso faz-se necessário o acompanhamento periódico dessas pessoas, através de consultas, exames e realização de rastreamento de quadros de hiperglicemia crônica, visando prevenir e reduzir a incidência das doenças oculares, DV grave e cegueira irreversível.

\section{REFERÊNCIAS}

1. Mosquera CFF, Souza SC, Souza WC, Nascimento ACSG, Schlesener AH. Avaliação do equilíbrio estático em crianças com deficiência visual (DV). Revista InCantare. 2018 [cited 2019 jun 21]; 9(1):9-23. Available from: http://periodicos.unespar.edu.br/index.php/incantare/article/view/2337/pdf_86.

2. Avila M, Alves MR, Nishi M. As condições de saúde ocular no Brasil. São Paulo: Conselho Brasileiro de Oftalmologia; 2015 [cited 2019 Mar 04]. Available from: http://www.cbo.net.br/novo/publicacoes/Condicoes_saude_ocular_IV.pdf.

3. Bourne RRA, Flaxman SR, Braithwaite T, Cicinelli MV, Das A, Jonas JB, et al. Magnitude, temporal trends, and projections of the global prevalence of blindness and distance and near vision impairment: a systematic review and meta-analysis. Lancet Glob Health. 2017 [cited 2019 Jul 29]; 5(9):e888-e97. Available from: https://pure.qub.ac.uk/portal/files/134300690/Magnitude_temporal_trends_and_projections_of_the_global.pdf.

4. Safi H, Safi S, Hafezi-Moghadam A, Ahmadieh H. Early detection of diabetic retinopathy. Surv. Ophthalmol. 2018 [cited 2019 Ju 23]; 63(5):601-8. Available from: http://dx.doi.org/10.1016/j.survophthal.2018.04.003. 
5. International Diabetes Federation (IDF). IDF Diabetes Atlas, 8th edn. Brussels, Belgium: International Diabetes Federation; 2017 [cited 2019 Mar 04]. 46p. Available from: https://www.idf.org/e-library/epidemiology-research/diabetes-atlas.

6. Brito ES, Pinto MH, Beretta D, Pompeo DA, Brito TJF, Czorny RCN. Clinical and sociodemographic profile of patients with vision disorders and diabetes mellitus. Revista de Enfermagem do Centro-Oeste Mineiro. 2019 [cited 2020 Mar 08]; 9(3475):1-10. DOI: https://doi.org/10.19175/recom.v9i0.3475.

7. Sociedade Brasileira de Diabetes (SBD). Diretrizes da Sociedade Brasileira de Diabetes: 2017-2018. São Paulo: Editora Clannad; 2017 [cited 2019 Mar 10]. Available from: https://www.diabetes.org.br/profissionais/images/2017/diretrizes/diretrizes-sbd2017-2018.pdf.

8. American Diabetes Association. 2. Classification and diagnosis of diabetes: Standards of Medical Care in Diabetes-2018. Diabetes Care. 2018 [cited 2019 Mar 04]; 41(Suppl 1):S13-27. Available from: http://care.diabetesjournals.org/content/diacare/41/Supplement_1/S13.full.pdf.

9. Liu YC, Wilkins M, Kim T, Malyugin B, Mehta JS. Cataracts. Lancet. 2017 [cited 2019 Jul 29]; 390:600-12. Available from: https://www.sciencedirect.com/science/article/pii/S0140673617305445.

10. Secretaria de Direitos Humanos da Presidência da República ( $\mathrm{Br}$ ), Secretaria Nacional de Promoção dos Direitos da Pessoa com Deficiência, Coordenação-Geral do Sistema de Informações sobre a Pessoa com Deficiência. Cartilha do Censo 2010: Pessoas com Deficiência. Brasília: SDH PR/SNPD; 2012 [cited 2019 Jun 09]. 32p. Available from: https://bibliotecadigital.mdh.gov.br/jspui/handle/192/754.

11. Lima LR, Funghetto SS, Volpe CRG, Santos WS, Funez MI, Stival MM. Quality of life and time since diagnosis of Diabetes Mellitus among the elderly. Rev. bras. geriatr. gerontol. 2018 [cited 2019 Jul 23]; 21(2):176-85. DOI: http://dx.doi.org/10.1590/198122562018021.170187.

12. Tóth G, Szabó D, Sándor GL, Nagy ZZ, Karadeniz S, Limburg H, et al. Diabetes and blindness in people with diabetes in Hungary. Eur J Ophthalmol. 2019 [cited 2019 Jul 29]; 29(2):141-7. DOI: https://doi.org/10.1177/1120672118811738.

13. Lartey SY, Aikins AK. Visual impairment amongst adult diabetics attending a tertiary outpatient clinic. Ghana Med. J. 2018 [cited 2019 Jul 29]; 52(2):84-7. DOI: http://dx.doi.org/10.4314/gmj.v52i2.4.

14. Ramos AL, Bastar PAG, Lansingh VC, Gomez JAR, Fragoso VV, Arellano FAS, et al. Rapid assessment of avoidable blindness: Prevalence of blindness, visual impairment and diabetes in nuevo leon, Mexico 2014. Ophthalmic Epidemiol. 2018 [cited 2019 Jul 29]; 25:5-6, 412-8. DOI: https://doi.org/10.1080/09286586.2018.1501498.

15. Magalhães EMA, Carvalho CVD, Santos JA, Correia IF, Reis JWS, Valença TDC, et al. Aspectos socioeconômicos, de condições de saúde e hábitos de vida de pessoas idosas portadoras de diabetes. Ciência \& Desenvolvimento-Revista Eletrônica da FAINOR. 2019 [cited 2019 Set 29]; 12(1):179-91. DOI: https://doi.org/10.11602/1984-4271.2019.12.1.12.

16. Srinivasan S, Raman R, Ganesan S, Roy R, Natarajan V, Sharma T. Four-year incidence and progression of visual impairment in a South Indian population with diabetes. Indian J. Ophthalmol. 2017 [cited 2019 Out 07]; 65(7):589-95. Available from: https://doi.org/10.4103/ijo.IJO_520_16.

17. Martínez-González O, Martínez-González A, Martínez-Rosabal S. Patients treated with catar act diagnosis in the Municipality of Naguanagua. Carabobo. Venezuela. Multimed. 2017 [cited 2019 Set 29]; 21(3):233-40. Available from: http://www.revmultimed.sld.cu/index.php/mtm/article/view/539.

18. Moura KL, Catão CDS, Lima RA, Cruz JB. Lifestyle and self-perception in health in the control $f$ Type 2 Diabetes Mellitus. Rev. Ciênc. Méd. Biol. 2019 [cited 2019 Jul 28]; 18(1):52-60. DOI: http://dx.doi.org/10.9771/cmbio.v18i1.28426.

19. Lavaju P, Shah S, Sharma S, Maskey R. Diabetes Mellitus and the risk of Primary open angle glaucoma. Nepal J. Ophthalmol. 2017 [cited 2019 Jul 29]; 9(1):17-23. DOI: https://doi.org/10.3126/nepjoph.v9i1.17526.

20. Rim TH, Lee SY, Bae HW, Seong GJ, Kim SS, Kim CY. Increased risk of open-angle glaucoma among patients with diabetes mellitus: a 10-year follow-up nationwide cohort study. Acta Ophthalmol. 2018 [cited 2019 Jul 29]; 96:e1025-e30. DOI: https://doi.org/10.1111/aos.13805.

21. Gomes AR, Santos L. Prevalence of complications of diabetes mellitus in patients in the Santo Tirso/Trofa Group of Health Centres: a descriptive study. Rev. Port. Med. Geral Fam. 2017 [cited 2019 Set 08]; 33(4):252-60. Available from: http://www.scielo.mec.pt/scielo.php?script=sci_arttext\&pid=S2182-51732017000400003\&lng=pt.

22. Yau JW, Rogers SL, Kawasaki R, Lamoureux EL, Kowalski JW, Bek T, et al. Global prevalence and major risk factors of diabetic retinopathy. Diabetes care. 2012 [cited 2019 Set 29]; 35:556-64. DOI: http://dx.doi.org/10.2337/dc11-1909.

23. Matias COF, Matias COF, Alencar BR. Qualidade de vida em idosos portadores de Diabetes Mellitus Tipo 2 atendidos em Unidades Básicas de Saúde de Montes Claros/MG. Rev. bras. qual. vida. 2016 [cited 2019 Mar 12]; 8(2):119-29. Available from: https://periodicos.utfpr.edu.br/rbqv/article/view/3841/2782.

24. Oliveira BG, Bomfim ES, Ribeiro IJS, Carmo EA, Boery RNSO, Boery EN. Factors associated with the quality of life of individuals affected by diabetes mellitus. Rev. baiana enferm. 2017 [cited 2019 Set 29]; 31(4):e21481. DOI: https://doi.org/10.18471/rbe.v31i4.21481.

25. Liu Y, Song Y, Tao L, Qiu W, Lv H, Jiang X, et al. Prevalence of diabetic retinopathy among 13473 patients with diabetes mellitus in China: a crosssectional epidemiological survey in six provinces. BMJ Open 2017 [cited 2019 Out 07]; 7:e013199. DOI: https://doi.org/10.1136/bmjopen-2016-013199.

26. Amaral RT, Barbosa AM, Teixeira CC , Brandão LGVA, Afonso TC, Bezerra ALQ, et al. Knowledge of diabetics about disease and self-care. Rev. enferm. UFPE on line. 2019 [cited 2019 Mar 13]; 13(2):346-52. Available from: https://periodicos.ufpe.br/revistas/revistaenfermagem/article/view/239077/31283. 
27. Liew G, Michaelides M, Bunce C. A comparison of the causes of blindness certifications in England and Wales in working age adults (16-64 years), 1999-2000 with 2009-2010. BMJ Open. 2014 [cited 2019 Out 07]; 4(2):e004015. DOI: https://doi.org/10.1136/bmjopen-2013-004015.

28. Gale R, Scanlon PH, Evans M, Ghanchi F, Yang Y, Silvestri G, et al. Action on diabetic macular oedema: achieving optimal patient management in treating visual impairment due to diabetic eye disease. Eye (Lond). 2017 [cited 2019 Out 07];31(S1):S1-20. DOI: https://doi.org/10.1038/eye.2017.53. 